\title{
Article
}

\section{Multi-donor Fecal Microbial Transplantation for Critically Ill Patients: Rationale and Standard Operating Procedure}

\author{
Veronika Řehořováa ${ }^{*}$, Ivana Cibulková $^{2^{*}}$, Hana Soukupováa ${ }^{3}$ and František Duška ${ }^{1}$
}

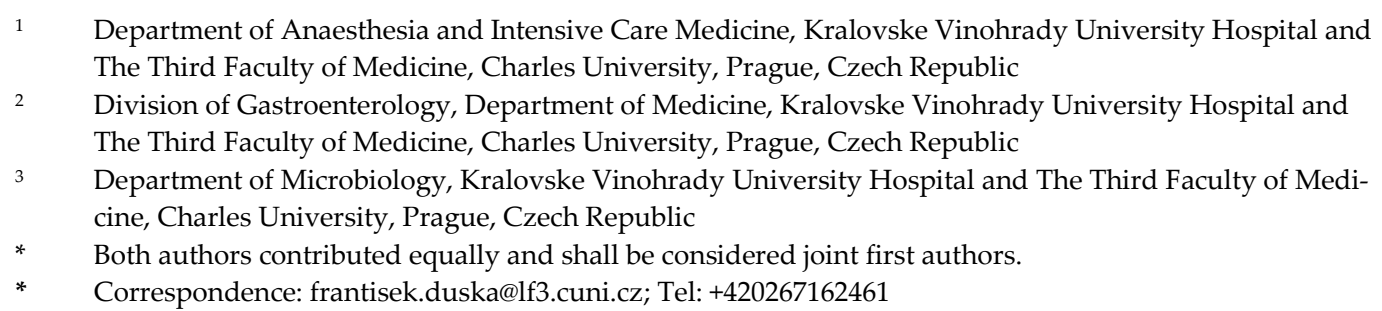

\begin{abstract}
Patients in the intensive care unit often lose a considerable fraction of their gut microbiome due to exposure to broad-spectrum antibiotics and other reasons. Dysbiosis often results in prolonged diarrhea and increase occurrence of multi-drug resistant pathogens in the colon with clinical consequences not yet well understood. Restoring the microbiome by faecal microbial transplantation (FMT) is a plausible therapeutic possibility, so far only documented in case reports and case series using very heterogeneous methodologies. Before FMT with critically ill patients can be tested in randomized controlled trials, there is a burning need to describe a standardized operating procedure (SOP) of the whole process, respecting the specifics of the critically ill population, such as the risk of the disrupted intestinal barrier and time-critical nature of the procedure. We describe the SOP that has been developed for experimental use in critically ill patients by a multidisciplinary team of intensivists, gastroenterologists, and microbiologists based on feedback from regulatory authority (State Institue of Drug Control of the Czech Republic). The hallmarks of these SOPs are multi-donor freshly frozen transplants guaranteed for 2 months consisting of 7 aliquots from 7 unrelated healthy donors and administered by a rectal tube. In this paper we discuss the rationale for this SOP and the process of its development in detail and release the full proposed SOP is in the form of an online appendix.
\end{abstract}

Keywords: faecal microbial transplantation; critically ill patients; standard operating procedure; diarrhea

\section{Introduction}

Although the sayings on the connection between the proper function of the gastrointestinal tract (GIT) and longevity have been known for decades, a full scientific description of such claims based on scientific evidence is yet to be made. Lumen inhabiting microorganisms (termed "microbiota") together with their metabolic products in the specific host environment (termed "microbiome") are indispensable for gastrointestinal physiology. The individual specifics of microbiome composition are determined by multiple factors, where genetics, early exposure to microorganisms (mainly transport from mother during birth), environment, and diet are considered the dominant ones[1,2]. Due to the microbiome's vast complexity, it is being considered as a separate organ by some researchers[3]. The recent progress in 16S rRNA sequencing brought about a proof that the microbiome composition of each individual is unique to such an extent, that it may be used in forensic disciplines[4,5]. This enormous microbiome diversity is pivotal to maintaining homeostasis[6,7]. In turn, a reduction of microbiome diversity (termed "dysbiosis") results in immune system dysfunction[8,9]. The association between altered microbiome and diseases has already been confirmed for a range of gastrointestinal tract diseases (eg. inflammatory 
bowel disease [10], irritable bowel syndrome - IBS[11], colorectal carcinoma[12]), gastric carcinoma, and liver disease[13]) as well as for metabolic syndrome [13], gout[14,15], neurodegenerative [16], autoimmune (rheumatoid arthritis, ankylosing spondylitis, lupus, sclerosis multiplext[14,15]) or psychiatric diseases [17], including depression[18].

Although the complex nature of these associations is yet to be understood, it is likely that two-way communication between microbiota and immune system exists [19] and is mediated by microbial-synthesized metabolites regulating host neuroimmune-inflammatory axes that could physiologically link gut with other organ systems [10]. Details of the interplay are summarized in a paper by Cibulkova et al. in this issue [65].

\section{Dysbiosis and Diarrhoea in Intensive Care Patients}

A recent study in patients admitted to intensive care showed, that $90 \%$ of intestinal microflora is lost during the first 6 hours of ICU stay[20]. The most common symptom of dysbiosis is diarrhea, which may not only complicate ICU stay by threatening skin surfaces, but also alter dignity and worsen the prognosis of ICU patients [21,22]. The main risk factors of diarrhea in ICU patients are antibiotics therapy, enteral nutrition[23], proton pump inhibitors (PPI) therapy[24], the severity of underlying disease[25], and history of recent surgery[26]. As approximately $70 \%$ of ICU patients worldwide are treated with antibiotics [27] and 5-25\% of those develop diarrhea [28], antibiotics are indeed the leading cause of diarrhea in ICU. On the other hand, the risk of diarrhea and its negative consequences are among the drivers of antibiotic stewardship programs [21,22]. In addition to this, the adoption of routine ICU care bundles has been suggested to reduce dysbiosis and diarrhea [29]. Dysbiosis is also linked to changes in intestinal barrier permeability and possible immune dysregulation and organ failures, [30,31], and also increased rate of colonization with multi-drug resistant pathogens.

\section{Faecal Microbial Transplantation: Defining the Needs of Standard Operating Proce- dure}

Faecal microbial transplantation (FMT sometimes termed "bacteriotherapy") is the transfer of stool preparation from a healthy donor (or donors) into the gastrointestinal tract of the recipient to treat or prevent disease[32]. The procedure aims to restore a healthy and diverse microbiome. Animal studies indicate that enteral nutrition or probiotics alone are not sufficient to restore commensal colonization of intestinal crypts [33,34]. Apart from induction the healthy and diverse bacteria [35], FMT also introduces dead bacteria, viruses, fungi, microbial peptides, and metabolites, as well as intact desquamated donor colonocytes, which all contribute to FMT effects [36,37, 38]. FMT triggers the release of antimicrobial factors, modulates the proliferation of immune cells, stimulates mucus formation and immune globulin A synthesis, inhibits activation of nuclear factor Kappa B in inflammatory epithelial cells, and increases mucin secretion, which in turn may restore the epithelial barrier and intestinal function[32].

FMT is not a new method, its first use was documented in China in $4^{\text {th }}$-century AD, where Ge Hong used a human stool to treat food poisoning or severe diarrhea[35]. In World War II, German soldiers were eating camel excrements as a remedy for bacterial dysentery during the Battle of Africa. In standard Western medicine, FMT was first used in 1958 as a therapy for pseudomembranous colitis. The method gradually became a wellestablished treatment for recurrent and refractory Clostridioides difficile infection (CDI)[36]. Along with the buildup of epidemiological evidence of an association between dysbiosis and a range of diseases, FMT has been considered a potential treatment beyond recurrent CDI[37]. Clinical trials have been published for the use of FMT in inflammatory bowel disease [38-40], and primary infection with Clostridioides difficile [41], and research is underway for Parkinson disease (NCT04854291), amyotrophic lateral sclerosis (NCT03766321), Alzheimer disease (NCT03998423, [42]). Given the rapid onset of dysbiosis in ICU patients and probable links with diarrhea, immune dysregulation, intestinal barrier disruption, multi-organ failure and MDR infections, early restoration of the 
microbiome through FMT may bring substantial benefit to ICU patients. On the other hand, introducing a surge of living microbes into the intestine of a very sick patient with dysregulated immunity and altered intestinal epithelial barrier function can bring about significant risks, mainly of bacterial translocation, sepsis, or worsening systemic inflammation - in addition to other known risks of FMT in noncritically ill population, including the introduction of MDRO from donor to the recipient [43] and death [44]. To date, only a case report and small case series have been published[45-48], and albeit their unequivocally positive results in treating diarrhea and improving overall patients' conditions are encouraging, these studies may be subjected to publication and attribution biases. There is a burning need for robust prospective, randomized controlled trials to evaluate the FMT safety and efficiency in this vulnerable patien's population.

Indeed, performing high-quality studies compliant with good clinical practice principles requires the approval of the respective regulatory authorities. These may feel uneasy to approve and oversee trials on FMT, particularly in vulnerable populations such as critically ill patients, e.g. due to the difficulties of standardization of the investigational product. In addition, logistical challenges, and the feasibility of FMT in a specific environment of ICU must be considered, too. For example, the State Institute for Drug Control (SUKL) of the Czech Republic decided in 2019 to consider FMT transplant a drug, and decided to apply the same principles of regulation and oversight as is in place for drug trials. Consequently, standard operating procedures (SOPs) are required for donor selection, transplant preparation, and recipient monitoring, ensuring the safety and reproducibility of FMT in clinical trials. In collaboration with the regulatory authority of one European Union country (SUKL), we have developed such an SOP, which we describe and explain below. Although directly applicable in only one EU country and only in the context of a clinical trial, we believe that it can be useful in its current form or after minor modification in other countries too, and help to standardize FMT procedure in clin ical trials in critically ill patients.

\section{Standard Operating Procedure of Faecal Microbial Transplantation in Intensive Care Unit Patients}

The full text of SOPs can be found in Supplementary Digital Content 1. There we describe the development of a standard operating procedure for multi-donor faecal microbial transplantation in critically ill patients, starting from donor selection and finishing with post-procedure recipient care. In summary, our SOP recommends the use of a multidonor transplant consisting of seven $50 \mathrm{mlL}$ aliquots from seven donors. The aliquots are prepared from healthy unrelated volunteers, who regularly donate. In addition to standard guidelines-driven precautions[49] in donors to prevent transmissible infection, the deep-frozen transplant mixed with glycerol is quarantined for at least 2 months before use. The transplantation itself is performed by a semi-rigid rectal irrigation tube in a patient positioned to the left semi lateral Trendelenburg's position for 15 minutes and then in the right semi lateral Trendelenburg's position for another 15 minutes. This ensures the distribution of the transplant well throughout the length of the colon (Figure 1). This recommendation has been developed as a modification of existing guidelines for the use of FMT to eradicate Clostridioides difficile in other than intensive care settings [ 50,51]. All modifications and their justifications are described below. 


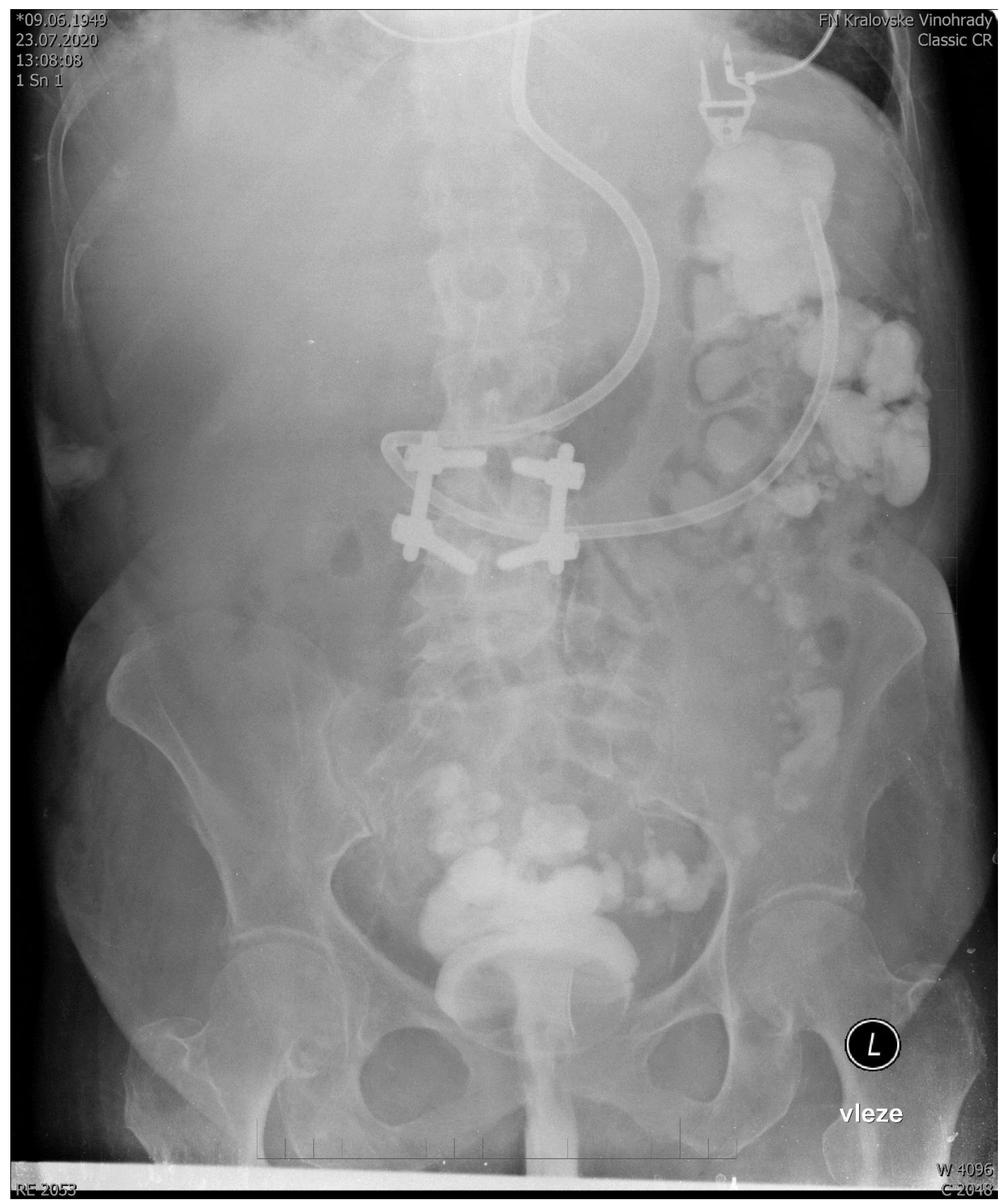

Figure 1. Abdominal $X$ ray showing the spread of $350 \mathrm{ml}$ transplant mixed with $5 \mathrm{ml}$ of contrast agent 15 mins after administration to a sedated ventilated patient via semirigid rectal tube. Faecal derivation system (Flexiseal (C) has been clamped immediately after administration with the rectal balloon inflated being clearly visible on the image. Note: The addition of a contrast agent is normally not a part of FMT and it should be noticed that there are no robust data on how FMT without contrast agent spreads after rectal administration.

\section{Donor selection and rationale for multi-donor strategy}

Appropriate donor selection is the key condition determining the FMT success[52]. The success rate is generally greater in FMT from a family member compared to an unrelated donor (93\% vs 84\%) [53] or from so-called "superdonors" with known high microbiome diversity[52,54]. It seems that the success of FMT procedure depends on the 
interaction (or rather compatibility) between the recipient's and donor's microbiome[55,56]. In theory, an ideal donor should have their microbiome as similar as possible to the premorbid microbiome of the recipient[57], or at least diverse enough that the mix certainly contains bacteria that the recipient will accept[51,55]. This idea led to concepts of faecal autotransplantation[58], a superdonor [54] or a multi-donor transplantation [59,60]. Multi-donor means mixing frozen-thawed small aliquots of stool from multiple donors into one graft for one recipient. We have adopted a multi-donor strategy for the use in the critically ill for the following reasons:

- Frozen samples are readily available and allow the best timing for the patient and staff regardless of donors' defecation habits. No need to exclude a large proportion of critically ill patients without relatives eligible for and willing to donate stool.

- Increase microbiome diversity as compared to a single donor

- Possibility of some degree of standardization, because aliquots from the same donors yield grafts of very similar composition

- Cost-effectiveness and affordability as compared to commercial preparations from super donors

As a downside, the multi-donor strategy increases the risk of transmission of infectious diseases that have escaped thorough screening[49]. It should be stressed that the ultimate responsibility for donor acceptance or rejection is with the physician examining them. The battery of tests represent the mandatory minimum, that can be extended if needed. Yet there always will be infections that are potentially transferable and are not investigated and the risk of those should be mentioned in the informed consent. The 2months graft quarantine with a repeated examination of the donor aims to minimize this risk. The number of aliquots from 7 donors has been determined by the convenience of practical $50 \mathrm{~mL}$ size aliquots and the required amount of transplant ( $350 \mathrm{~mL}$, see below).

\section{Donor stool processing and sample preparation}

Notably, among numerous guidelines and protocolson FMT, there is large heterogeneity in the process of sample preparation and often these important details are not reported at all. We recommend the use of normal saline as opposed to sterile water as it provides a better outcome [53]. Similarly, we have chosen $350 \mathrm{~mL}$ volume as a compromise between $500 \mathrm{~mL}$ that demonstrated a 97\% success rate, and $200 \mathrm{~mL}$ successful in 80 $\%$ of cases $[49,53]$. The use of frozen transplants has been chosen for the practical and safety reasons discussed above. It seems that $<12$ months of freezing does not significantly alter FMT efficacy [44], but further durability studies were requested by the SUKL.

\section{Southern way of administration}

With regards to the way of administration, various methods were considered. The lower GI or "Southern" route (via colonoscopy or retention enema) has been found more efficient in microbiome restoration compared to the upper GI "Nothern" routes (esophagogastroduodenoscopy, nasogastric, nasojejunal tube, poop pills)[61]. Also, we considered upper GI administration of faecal material into the small intestine with potentially disrupted epithelial barrier too risky. Of particular concern were aspiration, a recognized complication of the upper GI route [62]. Moreover, in a survey, the majority of ICU nurses considered the administration of faecal material into the colon more acceptable for them as opposed to nasogastric administration (Iva Havirova thesis, unpublished). Given the logistic issues with the availability of endoscopists and considerable risks of endoscopy[63], we have chosen administration by an enema, despite its lower efficiency, compared to colonoscopy. With appropriate patient positioning, we found graft distribution throughout the colon acceptable, reaching the ascendent colon (Figure 1). Indeed, this case-based anecdotal data are far from conclusive with regards to FMT spread and effect size. To maximize engraftment, we recommend performing FMT no sooner than 48 hours after the last dose of antibiotics. 


\section{Safety Considerations and Measures to Maximise Safety for Recipients}

Critically ill patients represent a very specific and vulnerable patient population, in which a normally very safe procedure[32] with only mild and self-limiting safe effects[64] can have additional serious risks that might outweigh benefits. In theory, the bacterial load could lead to sepsis and worsen organ failure. This is why we recommend thorough patient investigation and monitoring before, during, and after the procedure, on top of the safety precautions described above. So far published case reports and case series do not report any serious adverse and only one death in 31 patients (See Table 3 of Cibulkova et al. [65]), but this can be heavily biased as only successful procedures may have been selected for publication. Until more data is gained from randomized controlled trials, we only recommend lower GI administration of FMT to critically ill patients either for established indications in non-critically ill populations (such as in C. dif. infection) or limiting the study population for new indications (eg. antibiotic-associated diarrhea) to hemodynamically stable patients, do not have perforated viscus, recent colorectal surgery (less than 3months) or severe neutropenia (less than 500 per microliter). In addition to continuous monitoring of vital functions, which is mandatory in ICU, we recommend routine blood cultures be taken 3 hours post-FMT plus whenever there are signs of an inflammatory response (such as rigors or fever). Only when initial clinical trials yield more safety data, we recommend expanding research beyond and further. This SOP aims to make FMT in critically ill patients as safe as possible, but it should be stressed that good quality data on FMT safety in critically ill patients are still not available.

\section{Conclusion}

Critically ill patients often suffer from rapid-onset intestinal dysbiosis resulting in prolonged diarrhea and potentially other adverse consequences. Restoration of the physiological microbiome through FMT has been proven safe and effective in a range of conditions outside the intensive care unit. Despite biological plausibility and strong collateral evidence, there is no data on the safety and efficacy of FMT to treat dysbiosis-related conditions in critically ill patients. We have developed a standard operating procedure for the use of FMT in critically ill patients. The hallmark of the methodology is the use of frozen multi-donor transplant quarantined for 3 months and administered by an enema to carefully monitored recipient.It should be stressed that FMT in critically ill patients should only be used in the context of randomized controlled trials. A multi-disciplinary approach is warranted to the design and conduct of clinical trials in this exciting field.

Declarations

Authors declare no conflict of interest.

Authors contribution. VR and IC drafted first version of the manuscript, which was critically revised by HS and FD, the latter finalising the draft. All authors read and approved the final form of the manuscript and agreed to submit it.

\section{References}

1. Human, T.; Project, M. Structure , Function and Diversity of the Healthy Human Microbiome. Nature 2012, 486, 207214, doi:10.1038/nature11234.

2. $\quad$ Haro, C.; Garc, S.; Alcala-diaz, J.F.; Delgado-lista, J.; Perez-martinez, P.; Oriol, A.; Camargo, A.; Perez-jimenez, F. The Gut Microbial Community in Metabolic Syndrome Patients Is Modified by Diet. The Journal of Nutritional Biochemistry 2015, doi:10.1016/j.jnutbio.2015.08.011.

3. Sender, R.; Fuchs, S.; Milo, R. Revised Estimates for the Number of Human and Bacteria Cells in the Body. 2016, 1-14, doi:10.1371/journal.pbio.1002533. 
4. $\quad$ Pei, A.Y.; Oberdorf, W.E.; Nossa, C.W.; Agarwal, A.; Chokshi, P.; Gerz, E.A.; Jin, Z.; Lee, P.; Yang, L.; Poles, M.; et al. Diversity of 16S RRNA Genes within Individual Prokaryotic Genomes . 2010, 76, 3886-3897, doi:10.1128/AEM.0295309.

5. Johnson, J.S.; Spakowicz, D.J.; Hong, B.; Petersen, L.M.; Demkowicz, P.; Chen, L.; Leopold, S.R.; Hanson, B.M.; Agresta, H.O.; Gerstein, M.; et al. Evaluation of 16S RRNA Gene Sequencing for Species and Strain-Level Microbiome Analysis. Nature Communications 1-11, doi:10.1038/s41467-019-13036-1.

6. Moens, E. Epithelial Barrier Biology: Good Fences Make Good Neighbours. 2011, 1-8, doi:10.1111/j.13652567.2011.03506.x.

7. Golovkina, T.; Bogatyrev, S.R.; Ismagilov, R.F.; Pamer, E.G. Commensal Symbiosis in Sickness. 2015, 514, 638-641, doi:10.1038/nature13823.Rapid.

8. $\quad$ Blaser, M.J. Harnessing the Power of the Human Microbiome. 2010, 107, 6125-6126, doi:10.1073/pnas.1002112107.

9. $\quad$ Althani, A.A.; Marei, H.E.; Hamdi, W.S.; Nasrallah, G.K.; Zowalaty, M.E.E.L.; Khodor, S.A.L.; Al-asmakh, M.; Abdelaziz, H.; Cenciarelli, C. Human Microbiome and Its Association With Health and Diseases. 2015, 1688-1694, doi:10.1002/jcp.25284.

10. Baumgart, D.C.; Carding, S.R. Gastroenterology 1 Infl Ammatory Bowel Disease : Cause and Immunobiology. 2007, 369, 1627-1640.

11. Ponnusamy, K.; Choi, J.N.; Kim, J.; Lee, S.; Lee, C.H. Microbial Community and Metabolomic Comparison of Irritable Bowel Syndrome Faeces Printed in Great Britain. 2011, 817-827, doi:10.1099/jmm.0.028126-0.

12. Arthur, J.C.; Arthur, J.C.; Perez-chanona, E.; Mühlbauer, M.; Tomkovich, S.; Uronis, J.M.; Fan, T.; Campbell, B.J.; Abujamel, T.; Dogan, B.; et al. Of the Microbiota. 2013, 120, 10-14, doi:10.1126/science.1224820.

13. Lau, E.; Carvalho, D.; Freitas, P. Gut Microbiota : Association with NAFLD and Metabolic Disturbances. $2015,2015$.

14. Wu, H.; Wu, E. In Immune Homeostasis and Autoimmunity @ 2012 Landes Bioscience . Do Not Distribute . 2012, 0976, doi:10.4161/gmic.19320.

15. Cianci, R.; Pagliari, D.; Piccirillo, C.A.; Fritz, J.H.; Gambassi, G. Editorial The Microbiota and Immune System Crosstalk in Health and Disease. 2018, 2018, 10-13.

16. Chapman, M.R.; Hufnagel, D.A. Disease to Dirt: The Biology of Microbial Amyloids. 2013, 9, 9-12, doi:10.1371/journal.ppat.1003740.

17. Hsiao, E.Y.; Mcbride, S.W.; Hsien, S.; Sharon, G.; Hyde, E.R.; Mccue, T.; Codelli, J.A.; Chow, J.; Reisman, S.E.; Petrosino, J.F.; et al. Microbiota Modulate Behavioral and Physiological Abnormalities Associated with Neurodevelopmental Disorders. Cell 2013, 155, 1451-1463, doi:10.1016/j.cell.2013.11.024.

18. Winter, G.; Hart, R.A.; Charlesworth, R.P.G.; Sharpley, C.F. Gut Microbiome and Depression : What We Know and What We Need to Know. 2018, 29, 629-643.

19. Microbiota, T.G. Interactions Between the Microbiota and the Immune System. 2012, 1268-1274.

20. Mcclave, S.A.; Patel, J.; Bhutiani, N. Should Fecal Microbial Transplantation Be Used in the ICU ? 2018, 1-7, doi:10.1097/MCC.0000000000000489.

21. Bloos, F.; Thomas-rüddel, D.; Rüddel, H.; Engel, C.; Schwarzkopf, D.; Marshall, J.C. Impact of Compliance with Infection Management Guidelines on Outcome in Patients with Severe Sepsis : A Prospective Observational Multi-Center Study. 2014, doi:10.1186/cc13755.

22. Arulkumaran, N.; Routledge, M.; Schlebusch, S.; Lipman, J.; Morris, A.C. Antimicrobial - Associated Harm in Critical Care : A Narrative Review. Intensive Care Medicine 2020, doi:10.1007/s00134-020-05929-3.

23. Vieira, L.V.; Pedrosa, L.A.C.; Souza, V.S.; Paula, C.A.; Rocha, R. Incidence of Diarrhea and Associated Risk Factors in Patients with Traumatic Brain Injury and Enteral Nutrition. Metabolic Brain Disease 2018, 33, 1755-1760, doi:10.1007/s11011-018-0287-2. 
24. Buendgens, L.; Bruensing, J.; Matthes, M.; Dückers, H.; Luedde, T.; Trautwein, C.; Tacke, F.; Koch, A. Administration of Proton Pump Inhibitors in Critically Ill Medical Patients Is Associated with Increased Risk of Developing Clostridium Difficile-Associated Diarrhea. Journal of Critical Care 2014, 29, 696.e11-696.e15, doi:10.1016/j.jcrc.2014.03.002.

25. Kyne, L.; Sougioultzis, S.; Mcfarland, L. V; Kelly, C.P. U NDERLYING D ISEASE S EVERITY AS A M AJOR R ISK. 2014, 23.

26. Thibault, R.; Graf, S.; Clerc, A.; Delieuvin, N.; Heidegger, C.P.; Pichard, C. Diarrhoea in the ICU: Respective Contribution of Feeding and Antibiotics. Critical Care 2013, 17, 8-10, doi:10.1186/cc12832.

27. Vincent, J.; Marshall, J.; Anzueto, A.; Martin, C.D.; Gomersall, C. And Outcomes of Infection in Intensive Care Units. 2009, 302, 2323-2329.

28. Treatment and Prevention of Antibiotic Associated Diarrhea. 2000, 16, 521-526.

29. Mcclave, S.A.; Lowen, C.C.; Martindale, R.G. The 2016 ESPEN Arvid Wretlind Lecture : The Gut in Stress *. Clinical Nutrition 2017, 1-18, doi:10.1016/j.clnu.2017.07.015.

30. Klingensmith, N.J. T h e G u t a s t h e Mo t o r o f Multiple Organ Dysfunction in Critical Illness Sepsis MODS Gut Intestine Critical Illness. Critical Care Clinics 2015, doi:10.1016/j.ccc.2015.11.004.

31. Alverdy, J.C.; Krezalek, M.A. Collapse of the Microbiome, Emergence of the Pathobiome, and the Immunopathology of Sepsis. Critical Care Medicine 2017, 45, 337-347, doi:10.1097/CCM.0000000000002172.

32. Smits, L.P.; Bouter, K.E.C.; Vos, W.M. De; Borody, T.J.; Nieuwdorp, M. Therapeutic Potential of Fecal Microbiota Transplantation. Gastroenterology 2013, doi:10.1053/j.gastro.2013.08.058.

33. Zaborin, A.; Krezalek, M.; Hyoju, S.; Defazio, J.R.; Setia, N.; Belogortseva, N.; Bindokas, V.P.; Guo, Q.; Zaborina, O.; Alverdy, J.C. Critical Role of Microbiota within Cecal Crypts on the Regenerative Capacity of the Intestinal Epithelium Following Surgical Stress. 2021, 112-122, doi:10.1152/ajpgi.00294.2016.

34. Wischmeyer, P.E.; Mcdonald, D.; Knight, R. Role of the Microbiome, Probiotics , and 'Dysbiosis Therapy ' in Critical Illness. 2016, 347-353, doi:10.1097/MCC.0000000000000321.

35. Zhang, F. Should We Standardize the 1, 700-Year-Old Fecal Microbiota Transplantation? 2012, 1755, doi:10.1038/ajg.2012.251.

36. Drekonja, D.; Reich, J.; Gezahegn, S.; Greer, N.; Shaukat, A. Annals of Internal Medicine Fecal Microbiota Transplantation for Clostridium Difficile Infection. 2015, 162, doi:10.7326/M14-2693.

37. Choi, H.H.; Cho, Y. Fecal Microbiota Transplantation : Current Applications, Effectiveness, and Future Perspectives. 2016, 257-265.

38. Meyer, G.W.; Pinn, D.M.; Aroniadis, O.C. Are Not Related to Abnormal Motor Function Is Fecal Microbiota Transplantation the Answer for Irritable Bowel Syndrome? A Single-Center Response to Meyer and Reestablishment of the Intestinal Microbiota With Fecal Microbiota Resolves Sepsis and Diarrhea . 2014, 1831-1832, doi:10.1038/ajg.2014.319.

39. Rossen, N.G.; Fuentes, S.; Spek, M.J. Van Der; Tijssen, J.; Hartman, H.A.; Duflou, A.; Löwenberg, M.; Brink, G.R. Van Den; Elisabeth, M.H.; Vos, W.M. De; et al. Findings from a Randomized Controlled Trial of Fecal Transplantation for Patients with Ulcerative Colitis. Gastroenterology 2015, doi:10.1053/j.gastro.2015.03.045.

40. Cui, B.; Feng, Q.; Wang, H.; Wang, M.; Peng, Z.; Li, P.; Huang, G.; Liu, Z.; Wu, P.; Fan, Z.; et al. Fecal Microbiota Transplantation through Mid-Gut for Refractory Crohn's Disease: Safety, Feasibility and Efficacy Trial Results 1., doi:10.1111/jgh.12727.

41. Biotherapeutics, A.; Infection, C.I.; Survival, M. C or r e Sp Ondence Fecal Microbiota Transplantation for Primary Clostridium Difficile Infection. 2018, 26-28.

42. Segal, A.; Zlotnik, Y.; Moyal-Atias, K.; Abuhasira, R.; Ifergane, G. Fecal Microbiota Transplant as a Potential Treatment for Parkinson's Disease - A Case Series. Clinical Neurology and Neurosurgery 2021, 207, 106791, doi:https://doi.org/10.1016/j.clineuro.2021.106791. 
43. https://www.fda.gov/safety/medical-product-safety-information/fecal-microbiota-transplantation-safety-alert-riskserious-adverse-events-likely-due-transmission [laste accessed 24-Oct-2021]

44. DeFilipp Z, Bloom PP, Torres Soto M, Mansour MK, Sater MRA, Huntley MH, Turbett S, Chung RT, Chen YB, Hohmann EL. Drug-Resistant E. coli Bacteremia Transmitted by Fecal Microbiota Transplant. N Engl J Med. 2019 Nov 21;381(21):2043-2050. doi: 10.1056/NEJMoa1910437. Epub 2019 Oct 30.

45. Li, Q.; Wang, C.; Tang, C.; He, Q.; Zhao, X.; Li, N.; Li, J. Successful Treatment of Severe Sepsis and Diarrhea after Vagotomy Utilizing Fecal Microbiota Transplantation : A Case Report. 2015, 1-12, doi:10.1186/s13054-015-0738-7.

47. Wei, Y.; Yang, J.; Wang, J.; Yang, Y.; Huang, J.; Gong, H.; Cui, H.; Chen, D. Successful Treatment with Fecal Microbiota Transplantation in Patients with Multiple Organ Dysfunction Syndrome and Diarrhea Following Severe Sepsis. Critical Care 2016, 1-9, doi:10.1186/s13054-016-1491-2.

47. Trubiano, J.A.; Gardiner, B.; Kwong, J.C.; Ward, P.; Testro, A.G.; Charles, P.G.P. Faecal Microbiota Transplantation for Severe Clostridium Difficile Infection in the Intensive Care Unit. European Journal of Gastroenterology and Hepatology 2013, 25, 255-257, doi:10.1097/MEG.0b013e32835b2da9.

48. Wurm, P.; Spindelboeck, W.; Krause, R.; Plank, J.; Fuchs, G.; Bashir, M.; Petritsch, W.; Halwachs, B.; Langner, C.; Högenauer, C.; et al. Antibiotic-Associated Apoptotic Enterocolitis in the Absence of a Defined Pathogen: The Role of Intestinal Microbiota Depletion. Critical Care Medicine 2017, 45, e600-e606, doi:10.1097/CCM.0000000000002310.

9. Cammarota, G.; Ianiro, G.; Tilg, H.; Rajilić-Stojanović, M.; Kump, P.; Satokari, R.; Sokol, H.; Arkkila, P.; Pintus, C.; Hart, A.; et al. European Consensus Conference on Faecal Microbiota Transplantation in Clinical Practice. Gut 2017, 66, 569580, doi:10.1136/gutjnl-2016-313017.

51. Lee, C.H.; Steiner, T.; Petrof, E.O.; Smieja, M.; Roscoe, D.; Nematallah, A.; Scott Weese, J.; Collins, S.; Moayyedi, P.; Crowther, M.; et al. Frozen vs Fresh Fecal Microbiota Transplantation and Clinical Resolution of Diarrhea in Patients with Recurrent Clostridium Difficile Infection a Randomized Clinical Trial. JAMA - Journal of the American Medical Association 2016, 315, 142-149, doi:10.1001/jama.2015.18098.

51. Jiang, Z.D.; Ajami, N.J.; Petrosino, J.F.; Jun, G.; Hanis, C.L.; Shah, M.; Hochman, L. Alimentary Pharmacology and Therapeutics Randomised Clinical Trial: Faecal Microbiota Transplantation for Recurrent Clostridum Dif Fi Cile Infection - Fresh, or Frozen, or Lyophilised Microbiota from a Small Pool of Healthy Donors Delivered by Colonosc. 2017, 1-10, doi:10.1111/apt.13969.

52. Vermeire, S.; Joossens, M.; Verbeke, K.; Wang, J.; Machiels, K.; Sabino, J.; Ferrante, M.; Assche, G. Van; Rutgeerts, P.; Raes, J. Donor Species Richness Determines Faecal Microbiota Transplantation Success in Inflammatory Bowel Disease. Journal of Crohn's and Colitis 2016, 10, 387-394, doi:10.1093/ecco-jcc/jjv203.

53. Gough, E.; Shaikh, H.; Manges, A.R. Systematic Review of Intestinal Microbiota Transplantation ( Fecal Bacteriotherapy ) for Recurrent Clostridium Difficile Infection. 2011, 53, doi:10.1093/cid/cir632.

54. Wilson, B.C.; Vatanen, T.; Cutfield, W.S.; O'Sullivan, J.M. The Super-Donor Phenomenon in Fecal Microbiota Transplantation. Frontiers in Cellular and Infection Microbiology 2019, 9, 1-11, doi:10.3389/fcimb.2019.00002.

55. Kelly, C.R.; Kahn, S.; Kashyap, P.; Laine, L.; Rubin, D.; Atreja, A.; Moore, T.; Wu, G. Update on Fecal Microbiota Transplantation 2015: Indications, Methodologies, Mechanisms, and Outlook. Gastroenterology 2015, 149, 223-237, doi:10.1053/j.gastro.2015.05.008.

56. Moayyedi, P.; Surette, M.G.; Kim, P.T.; Libertucci, J.; Wolfe, M.; Onischi, C.; Armstrong, D.; Marshall, J.K.; Kassam, Z.; Reinisch, W.; et al. Fecal Microbiota Transplantation Induces Remission in Patients With Active Ulcerative Colitis in a Randomized Controlled Trial. Gastroenterology 2015, 149, 102-109.e6, doi:10.1053/j.gastro.2015.04.001.

57. Khanna, S.; Vazquez-Baeza, Y.; González, A.; Weiss, S.; Schmidt, B.; Muñiz-Pedrogo, D.A.; Rainey, J.F.; Kammer, P.; Nelson, H.; Sadowsky, M.; et al. Changes in Microbial Ecology after Fecal Microbiota Transplantation for Recurrent C. Difficile Infection Affected by Underlying Inflammatory Bowel Disease. Microbiome 2017, 5, 1-8, doi:10.1186/S40168017-0269-3. 
58. Taur, Y.; Coyte, K.; Schluter, J.; Robilotti, E.; Figueroa, C.; Gjonbalaj, M.; Littmann, E.R.; Ling, L.; Miller, L.; Gyaltshen, Y.; et al. Reconstitution of the Gut Microbiota of Antibiotic-Treated Patients by Autologous Fecal Microbiota Transplant. Science Translational Medicine 2018, 10, doi:10.1126/scitranslmed.aap9489.

59. Paramsothy, S.; Kamm, M.A.; Kaakoush, N.O.; Walsh, A.J.; Bogaerde, J. Van Den; Samuel, D.; Leong, R.W.L. Multidonor Intensive Faecal Microbiota Transplantation for Active Ulcerative Colitis : A Randomised Placebo-Controlled Trial. The Lancet 2017, 6736, 1-11, doi:10.1016/S0140-6736(17)30182-4.

60. Wilson, B.C.; Vatanen, T.; Jayasinghe, T.N.; Leong, K.S.W.; Derraik, J.G.B.; Albert, B.B.; Chiavaroli, V.; Svirskis, D.M.; Beck, K.L.; Conlon, C.A.; et al. Strain Engraftment Competition and Functional Augmentation in a Multi-Donor Fecal Microbiota Transplantation Trial for Obesity. 2021, 1-16.

61. Kassam, Z.; Lee, C.H.; Yuan, Y.; Hunt, R.H. Fecal Microbiota Transplantation for Clostridium Difficile Infection: Systematic Review and Meta-Analysis. American Journal of Gastroenterology 2013, 108, 500-508, doi:10.1038/ajg.2013.59.

62. Karakan, T. Fecal Microbiota Transplant in Immunocompromised Patients: Encouraging Results in a Vulnarable Population. Turkish Journal of Gastroenterology 2014, 25, 346, doi:10.5152/tjg.2014.0013.

63. Postigo, R.; Kim, J.H. Colonoscopic versus Nasogastric Fecal Transplantation for the Treatment of Clostridium Difficile Infection: A Review and Pooled Analysis. Infection 2012, 40, 643-648, doi:10.1007/s15010-012-0307-9.

64. van Nood, E.; Vrieze, A.; Nieuwdorp, M.; Fuentes, S.; Zoetendal, E.G.; de Vos, W.M.; Visser, C.E.; Kuijper, E.J.; Bartelsman, J.F.W.M.; Tijssen, J.G.P.; et al. Duodenal Infusion of Donor Feces for Recurrent Clostridium Difficile . New England Journal of Medicine 2013, 368, 407-415, doi:10.1056/nejmoa1205037.

65. Cibulková I, Řehořová V, Hajer J, Duška F. Fecal Microbial Transplantation in Critically Ill Patients-Structured Review and Perspectives. Biomolecules. 2021 Oct 4;11(10):1459. doi: 10.3390/biom11101459 